\title{
A Simple Method for Patterning Nanoparticles on Planar Surfaces
}

\author{
Getachew Tizazu \\ Department of Physics, Bahir Dar University, P.O. Box 3019, Bahir Dar, Ethiopia \\ Correspondence should be addressed to Getachew Tizazu; getacht@yahoo.com
}

Received 28 March 2019; Accepted 16 May 2019; Published 4 June 2019

Academic Editor: Andrey E. Miroshnichenko

Copyright (c) 2019 Getachew Tizazu. This is an open access article distributed under the Creative Commons Attribution License, which permits unrestricted use, distribution, and reproduction in any medium, provided the original work is properly cited.

This paper describes a simple method to pattern nanoparticles on planar surfaces using the antifouling property of poly(ethylene glycol) monolayers deposited from a solution on the native oxide of titanium. Atomic force microcopy was used to pattern the poly(ethylene glycol) monolayers producing protein active sites on the protein-resistant surface. Patterns with different sizes have been generated by shaving the monolayers with different repetitions. Friction force microscopy was used to image the patterns. The smallest patterns are $50 \mathrm{~nm}$ and the largest patterns are $500 \mathrm{~nm}$ at full width half maximum. The smallest pattern was produced with one shave, whereas the largest pattern was produced by shaving the monolayers 112 times. Protein-coated nanoparticles were immobilised on the shaved (protein active) part of the monolayers by dipping the patterned samples into a solution that contains $2 \%$ by volume protein-functionalized nanoparticles with a nominal diameter of $40 \mathrm{~nm}$. Atomic force microscopy was used to take a topographic image of the samples. The topographic image showed that the protein-functionalized nanoparticles were attached onto the shaved part of the substrate but not on the poly(ethylene glycol)-covered part of the substrate. The level of aggregation of the nanoparticles was also investigated from the topographic image. The section analysis of the topographic image of the nanoparticle patterns showed a height of $40 \mathrm{~nm}$ which proved that only a monolayer of particles were deposited on the shaved part of the monolayer.

\section{Introduction}

Controlling the nature and properties of surfaces is essential to position nanoparticles with desired geometries and dimensions $[1,2]$. Self-assembled monolayers, formed by the spontaneous assembly of adsorbates from dilute solution onto solid surfaces, have been used to control the properties of surfaces $[3,4]$. The surface properties of surfaces can also be altered by patterning of self-assembled monolayers. There are several patterning techniques for the production of multiple chemical components on solid surfaces [5]. For the micrometer-scale patterning, the most widely used techniques are photo patterning with a mask and microcontact printing $(\mu \mathrm{CP})$ which provides rapid reproduction of patterns with high fidelity $[6,7]$. The nanometer-scale patterning includes electron beam lithography, dip pen nanolithography, nanoshaving, and near-field optical techniques [8]. Wang et al. used dip pen for patterning of citrate-caped gold nanoparticles on solid surfaces [9]. Schmudde et al. deposited amino-functionalized silica nanoparticles on aldehyde-terminated self-assembled monolayers on gold surfaces. Consequently, the aminofunctionalized silica particles were used as templates for the subsequent self-assembly of a second type of nanoparticle, which is gold [10]. Khanh and Yoon used topologyassisted self-organisation of nanoparticles where photopatterned silicon wafers were used as substrates for organisation of nanoparticles in one dimension [11]. Mercado et al. used multiwall carbon nanotubes to guide the onedimensional arrangement of silver nanostructures [12]. However, all these techniques are expensive and time consuming since they involve many steps. Therefore, a low cost and easy method which enables to produce patterned nanoparticles on solid surfaces is essential. In this paper, we present a simple method that uses a one-step patterning of nanoparticles on solid surfaces using poly(ethylene glycol) monolayers formed on titanium oxide surfaces as a resist. Titanium was chosen as a substrate because it has many applications that range from nanomedicine to optoelectronics [13]. 
Poly(ethylene glycol) and related materials exhibit strong resistance to nonspecific adsorption of proteins because of steric repulsion between hydrated neutral poly(ethylene glycol) chains and the proteins $[14,15]$. Poly(ethylene glycol) deposited on titanium oxide using alkane phosphate as an anchoring group demonstrated good protein resistance. However, the use of alkane phosphate as an anchoring group showed poor stability [16]. Therefore, in this work, the use of silane as an anchoring group is preferred because of its stability due to the formation of two bonds between the molecules and an additional bond formed with the surface [2]. These intermolecular bonds can be used to form a laterally polymerised network resulting in an improved stability of the monolayers.

\section{Materials and Methods}

2.1. Materials. Titanium wires (99.9\%) were obtained from Goodfellow (Cambridge, UK). Ethanol (HPLC grade), hydrogen peroxide ( $30 \%$ by volume), and concentrated sulphuric acid (70\% by volume) were obtained from Fisher Scientific Ltd (Loughborough, UK). Poly(ethylene glycol)silane was purchased from Fluorochem Ltd (Derbyshire, UK). NeutrAvidin-labelled polystyrene nanospheres (2\% solid with a nominal diameter of $40 \mathrm{~nm}$ ) were purchased from Invitrogen Ltd (Paisley, UK). All glassware and substrates were cleaned using piranha solution (a mixture of $30 \% \mathrm{H}_{2} \mathrm{O}_{2}$ and $98 \%$ concentration sulphuric acid in the ratio $3: 7$ ) for $45 \mathrm{~min}$.

2.2. Preparation of Titanium Film. An Edwards Auto 306 vacuum evaporator fitted with quartz balance for thickness measurement was used during the deposition of titanium films. The vacuum was pumped down to $1 \times 10^{-7}$ mbar prior to evaporation. A $30 \mathrm{~nm}$ thick titanium films were prepared by evaporating titanium metal from a tungsten boat onto cleaned glass slides at a rate of $0.05 \mathrm{~nm} \cdot \mathrm{s}^{-1}$.

2.3. Preparation of Poly(ethylene glycol)-Silane Films. Titanium films were dipped into a $0.05 \mathrm{mM}$ poly(ethylene glycol)-silane solution in anhydrous toluene under nitrogen. The deposition was preceded for 24 hrs for both poly(ethylene glycol)-trichlorosilane and poly(ethylene glycol)silane solutions. The samples were ultrasonically cleaned in fresh toluene for $15 \mathrm{~min}$ and fresh ethanol for another $15 \mathrm{~min}$. They were cured in a vacuum oven at $120^{\circ} \mathrm{C}$ for $45 \mathrm{~min}$ to increase crosslinking, thereby increasing stability. In this experiment, two types of silane anchoring groups were used, namely, trichlorosilane and trimethoxysilane.

\subsection{Characterisation with Contact Angle Measurement.} To investigate the surface wet ability, static water contact angle measurement was taken by using a Rame-Hart model 100-00 contact angle ganiometer. Deionised water was used as a drop. The contact angle measurement was taken at five different points on the surface of the sample and then averaged. The poly(ethylene glycol)-silane monolayer coverage was calculated from the contact angle using [17]

$$
\begin{array}{r}
(1+\cos \theta)^{2}=f_{1}\left(1+\cos \theta_{1}\right)^{2}+f_{2}\left(1+\cos \theta_{2}\right)^{2}, \\
1=f_{1}+f_{2},
\end{array}
$$

where $\theta$ is the measured angle, $f_{1}$ is the area covered by titanium oxide, $\theta_{1}$ is the contact angle of bare titanium oxide, $f_{2}$ is the area covered by poly(ethylene glycol)-silane, and $\theta_{2}$ is the contact angle of the poly(ethylene glycol)-silane at full coverage.

2.5. Thickness Measurement. Horiba UVISEL Ellipsometry was used to determine the thickness and surface density of poly(ethylene glycol)-silane film formed on the titanium oxide. The refractive index of the poly(ethylene glycol)silane film was taken as 1.6. The average distance $(L)$ between grafted chains was estimated from the ellipsometric thickness using [18]

$$
L=\left(\frac{M}{h \rho N_{\mathrm{A}}}\right)^{1 / 2},
$$

where $M$ is the molecular weight (700), $h$ is the thickness, $\rho$ is the density of the dry poly(ethylene glycol) film $\left(1.08 \times 10^{-24} \mathrm{~g} / \mathrm{A}^{3}\right)$, and $N_{\mathrm{A}}$ is Avogadro's number.

The grafting density $(\sigma)$ which is used to estimate the orientation of the grafted polymers was calculated using

$$
\sigma\left(\frac{\text { chains }}{\mathrm{nm}^{2}}\right)=\frac{h \rho N_{\mathrm{A}}\left(\mu \mathrm{g} / \mathrm{cm}^{2}\right) \times 10^{-20}}{M} .
$$

The diameter of the hydrated molecules $\left(d_{\text {wet }}\right)$ was determined from the radius of gyration for a polymer molecule ( $R_{\mathrm{G}}=0.02 \mathrm{M}^{0.58}$, where $M$ is the molecular weight) using the relation $d_{\mathrm{wet}}=2 R_{\mathrm{G}}$.

2.6. Elemental Analysis. Surface analysis by X-ray photoelectron spectroscopy (XPS) was carried out using a Kratos Axis Ultra DLD X-ray photoelectron spectrometer equipped with a monochromatic Al-K $\alpha$ X-ray source in an ultrahigh vacuum environment.

2.7. Roughness Measurement. Nanoscope III multimode atomic force microscopes (Digital Instruments, Santa Barbra, CA) were used for characterising the film surfaces. Root mean square (rms) roughness was determined from a topographic image of the poly(ethylene glycol)-silane film.

2.8. Patterning. The Nanoscope III multimode atomic force microscopes fitted with cantilevers with a force constant of $40 \mathrm{~N} / \mathrm{m}$ was used for shaving the poly(ethylene glycol)-silane films. The load needed to cause plastic deformation of the sample is dependent on the radius of the tip (equation (6)). A smaller radius results in plastic deformation at relatively lower loads than a tip with larger radius [19]. The maximum pressure, $P$, the tip can apply on the sample is $3 / 2$ times the 
mean pressure (equation (4)). The depth, $h$, the tip can deform the substrate is given by equation (6) [20]:

$$
\begin{aligned}
& P=\frac{3}{2} \frac{F}{\pi R^{2}}, \\
& h=\frac{(F D)^{2 / 3}}{R^{1 / 3}},
\end{aligned}
$$

where

$$
D=\frac{3}{4}\left(\frac{1-\sigma^{2}}{E}-\frac{1-\sigma^{\prime 2}}{E^{\prime}}\right),
$$

where $F$ is the force applied, $R$ is the radius of the tip, $E$ and $E^{\prime}$ are Young's moduli, and $\sigma$ and $\sigma^{\prime}$ are Poisson's ratios of the tip (silicon nitride) and the substrate (titanium oxide), respectively.

Friction force microscopy has been used to image the patterned samples. In friction force microscopy, the apex of a sharp tip is brought into contact with a sample surface, and the lateral forces are recorded while tip and sample slide relative to each other. In this case, soft cantilevers with force constant $0.38 \mathrm{~N} / \mathrm{m}$ has been used not to scratch while imaging the surface.

\subsection{Site-Specific Attachment of Nanoparticles.} NeutrAvidin-coated nanoparticle attachment was carried out by immersing the patterned samples into a $0.1 \mathrm{M} 2-(\mathrm{N}-$ morpholino)ethanesulfonic acid (MES) buffer of $\mathrm{pH} 6.1$ that contains $10 \mu \mathrm{L}$ of the nanoparticle, for $1 \mathrm{hr}$. A topographic image was taken using atomic force microscopy after the samples were cleaned using ethanol. Figure 1 shows a schematic of the whole process in patterning proteinfunctionalized nanoparticles on planar surfaces.

\section{Results and Discussion}

Poly(ethylene glycol) monolayers were formed on the native oxide of titanium using two types of anchoring groups, trimethoxysilane and trichlorosilane. The wetting properties of both monolayers were investigated using contact angle measurement. The contact angle for poly(ethylene glycol)trichlorosilane was $40^{\circ}$ and for poly(ethylene glycol)trimethoxysilane was $34^{\circ}$. The contact angle measurement indicates that the surface of the titanium oxide changes from hydrophilic to hydrophobic since the unmodified titanium oxide surface showed a contact angle measurement of $5^{\circ}$. The contact angle of the poly(ethylene glycol)trichlorosilane films was greater than the contact angle of the poly(ethylene glycol)-methoxysilane due to the difference in the rate of reaction of the molecules with water. Trichlorosilanes hydrolyse and polymerise very quickly both in the solution and on the surface of the substrate in the presence of a trace of water which can cover the substrate more quickly than trimethoxy silanes which hydrolyse very slowly [21].

The surface coverage by the poly(ethylene glycol) monolayer was estimated from equation (1). The contact angle for the fully covered polyethylene monolayer was taken from previous works by Sharma et al. which is $42^{\circ}$ [22]. Using these data, poly(ethylene glycol)-trichlorosilane monolayer showed a $91 \%$ coverage while poly(ethylene glycol)methoxysilane coverage was only $67 \%$. From the contact angle measurement and coverage calculation, poly(ethylene glycol)-trichlorosilane demonstrated better hydrophobic surfaces with high water contact angles. Consequently, it was chosen for further studies. Water contact angle was also used to study the stability of the monolayer in aqueous solutions. The samples have been immersed in Milli- $Q$ water for a duration that ranges from 0 to 24 hours. The water contact angle decreases initially but stayed constant at $37^{\circ}$ afterwards. Most probably, at the beginning, molecules which were not bound strongly desorbed reducing the water contact angle; however, after the unbound molecules are desorbed, the water contact angle stayed constant which shows that the poly(ethylene glycol)-trichlorosilane films are robust due to networked nature of silane bonds with the native oxide and with each other. In addition, polyethylene glycol is well known for protecting the native oxide from water degradation [7]. Compared with phosphonic acid monolayers on the native oxide of titanium, poly(ethylene glycol)-trichlorosilane monolayers showed better stability in water [23]. Therefore, poly(ethylene glycol)-trichlorosilane-coated oxide surfaces may be preferred in fabricating devices which may be in contact with aqueous solution during application.

The thickness of the poly(ethylene glycol)-trichlorosilane film was measured using ellipsometry. The ellipsometric value of the poly(ethylene glycol)-trichlorosilane film thickness was $1.6 \mathrm{~nm}$. The result is consistent with literature values indicating the formation of only a monolayer [22]. However, the thickness is less than the length of a trans-extended chain which is greater than $3 \mathrm{~nm}$, indicating that the long axis of the poly(ethylene glycol) chains are not fully oriented perpendicular to the substrate pointing away from the surface (Figure 2(b)). The average distance $(L)$ between the poly(ethylene glycol) chains grafted to the surface was compared with the diameter of the hydrated chains to determine the close up of any open space. The diameter $(d)$ of the hydrated poly(ethylene glycol) chains was determined from $d=2 R_{\mathrm{G}}$, where $R_{\mathrm{G}}=0.02 \mathrm{M}^{0.58}$ is the radius of gyration, and the distance between the chains was determined from equation (2). If $d>2^{1 / 2} L$, then the chains overlap and close any open space. In this experiment, the diameter of the hydrated molecules was $1.3 \mathrm{~nm}$ and the distance between the chains was $0.73 \mathrm{~nm}$. Therefore, poly(ethylene glycol) chains overlap and close any open space.

The roughness of the monolayers was analysed from the topographic image taken by atomic force microscopy. The root mean square $\left(R_{\mathrm{rms}}\right)$ roughness of the poly(ethylene glycol)-silane monolayer was found to be $0.8 \mathrm{~nm}$ which is greater than the roughness of the substrate. The monolayer was less smooth than the substrate because of low grafting density. Less-dense monolayers are rougher than the highly dense and close-packed monolayers. The atomic force microscopy image also shows no aggregates which is a characteristic of homogenous monolayers (Figure 2(a)). From the ellipsometric film thickness measurement and atomic force topographic image, the film is modelled as disordered and collapsed (Figure 2(b)). 


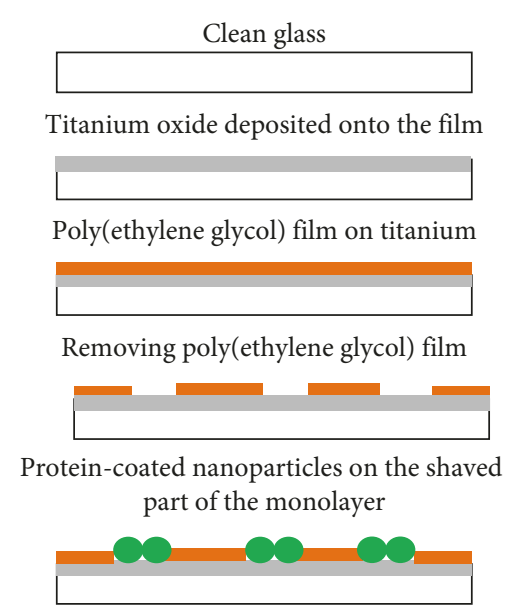

(a)

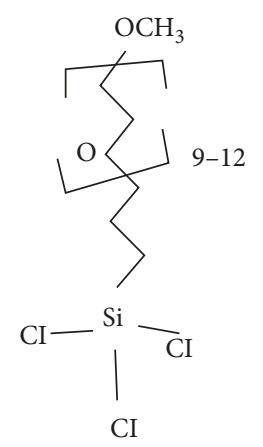

(b)

FIgUre 1: (a) Schematic showing the process of deposition of protein-coated nanoparticles. (b) Poly(ethylene glycol)-trichlorosilane molecule used for the formation of the monolayer.

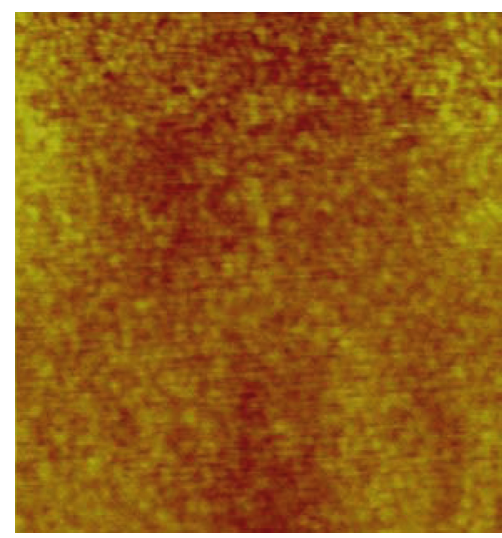

(a)

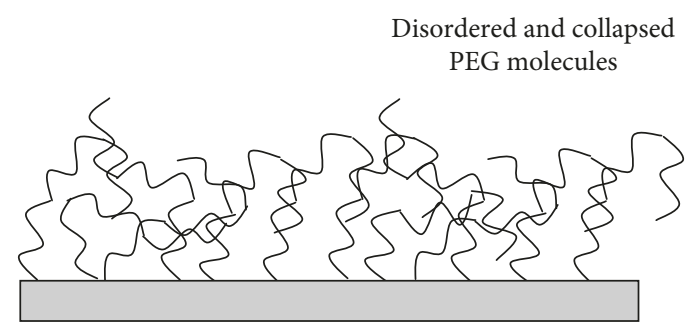

(b)

Figure 2: (a) A $1 \times 1 \mu \mathrm{m}^{2}$ atomic force microscopy topographic image of the poly(ethylene glycol)-silane monolayer on native oxide of titanium. (b) A schematic of the orientation of the poly(ethylene glycol) chains.

To determine the surface composition of the poly(ethylene glycol)-silane modified titanium oxide surfaces, elemental analysis was performed by X-ray photoelectron spectroscopy using a pass energy of $80 \mathrm{eV}$ and a take-off angle of $90^{\circ}$. The only elements that could be detected were carbon, oxygen, and titanium which were in agreement with the expectation. The high-resolution spectra for carbon have two $\mathrm{C} 1 \mathrm{~s}$ components at $286.6 \mathrm{eV}$ and $285 \mathrm{eV}$ which correspond to the ether and the aliphatic carbon atoms, respectively (Figure 3 ).

The poly(ethylene glycol)-silane monolayers have been patterned for guiding the assembly of protein-coated nanoparticles in one dimension. Nanopatterns were generated by shaving the monolayers using atomic force microscopy tip with force constant $12 \mathrm{~N} / \mathrm{m}$. The atomic force microscopy tip was scanned with different repetitions to produce different-sized patterns. During shaving, the force was set at $2000 \mathrm{nN}$ to ensure complete removal of the poly(ethylene glycol)-silane molecules. Figure 3 shows different-sized patterns produced by shaving the

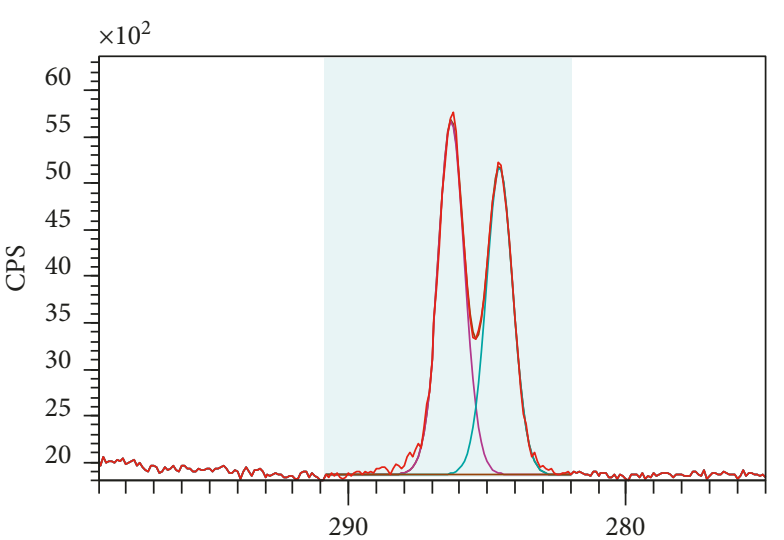

Figure 3: High-resolution X-ray photoelectron spectroscopy spectra of carbon.

poly(ethylene glycol)-silane monolayers with different scan repetitions. The shaved samples were sonicated in pure ethanol for $15 \mathrm{~min}$ to remove any fragmented molecules, and 


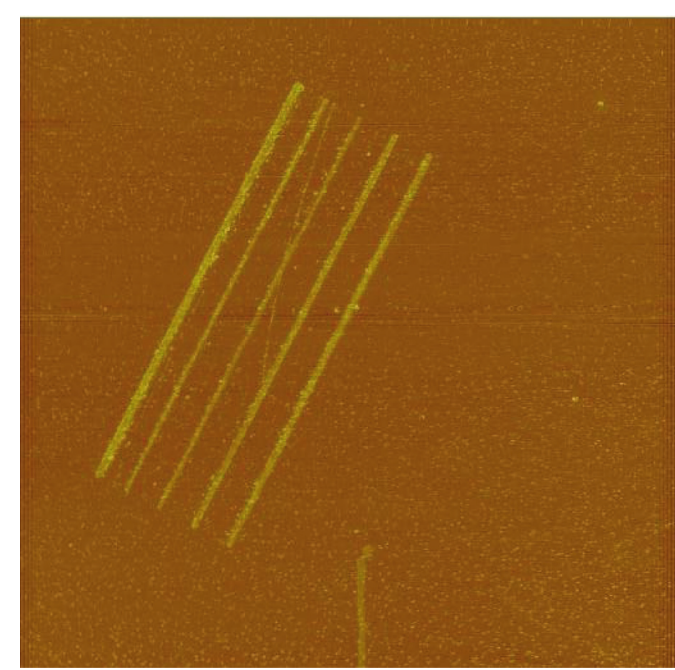

(a)

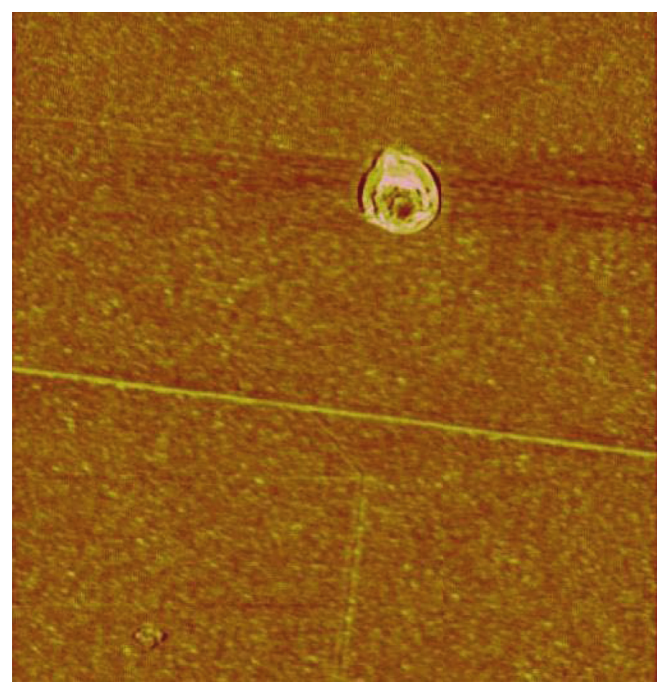

(c)

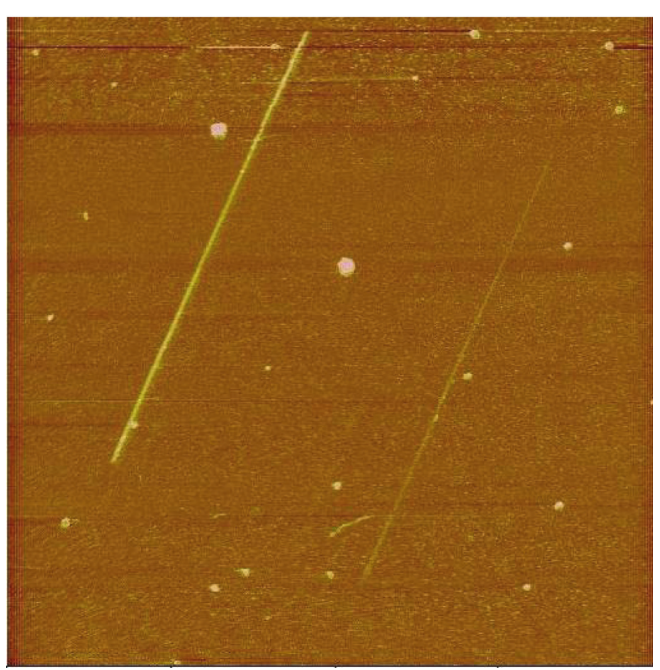

(b)

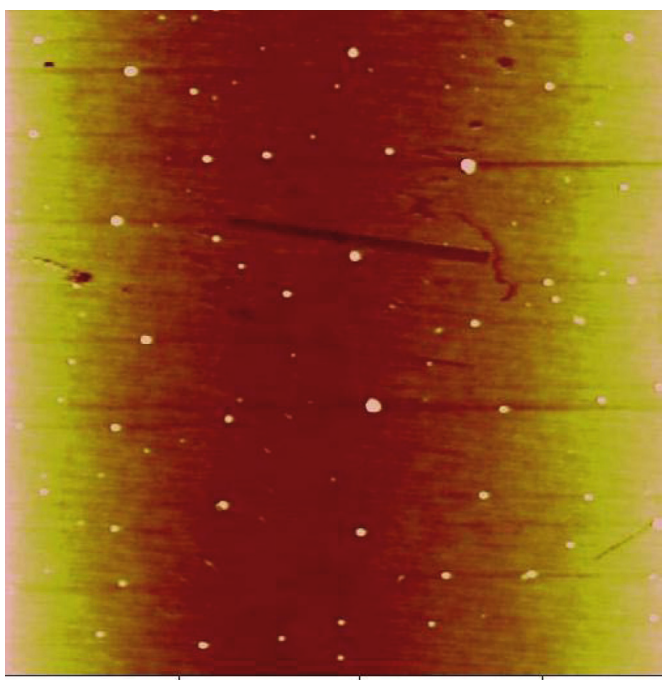

(d)

FIGURE 4: Friction force microscopy image of patterns produced by shaving the poly(ethylene glycol)-silane monolayer on titanium oxide: (a) different-sized patterns on the same sample (image size $20 \times 20 \mu \mathrm{m}^{2}, z$ range $0-1 \mathrm{~V}$ ); (b) $150 \mathrm{~nm}$ shaved part (image size $15 \times 15 \mu \mathrm{m}^{2}, z$ range $0-0.5 \mathrm{~V}$ ); (c) $50 \mathrm{~nm}$ shaved part (image size $5 \times 5 \mu \mathrm{m}^{2}$ ); (d) $35 \times 35 \mu \mathrm{m}^{2}$ topographic images of the scratches in (b).

then, friction force microscopy was used to image them. The shaved part showed brighter contrast than the unshaved part due to higher friction between the tip and the substrate. It is well known that self-assembled monolayers used for lubricating purposes. Therefore, the unshaved part appears less bright because of the lubrication effect of the monolayers. Figure 4(a) demonstrates the production of different-sized patterns on one sample by simply repeating the scan for wider patterns. Figure 4(b) shows a pattern with a width of $150 \mathrm{~nm}$ which was produced after scanning the sample for 112 times. Figure 4(c) shows a line of $50 \mathrm{~nm}$ which was produced after scanning the sample only once. Figure 4(d) shows a topographic image of the sample which shows that the depth of the scratches is ca $1.8 \mathrm{~nm}$. This depth is comparable with the ellipsometric thickness of the monolayer reported above. The difference between the film thickness and the depth of the shaved part is $0.2 \mathrm{~nm}$ which is much less than the roughness of the film. Therefore, it can be considered that the substrate is almost intact after shaving of the monolayers. The mechanism of shaving may be explained in relation to the bond energies of the molecules within the monolayer. The Si-O bond energy is $4.685 \mathrm{eV}$, and the $\mathrm{Si}-\mathrm{C}$ bond energy is $0.788 \mathrm{eV}$ [24]. Therefore, most probably, the removal of the monolayers occurs by cleavage of the Si-C bond since this is the weakest bond.

The substrate indentation by the tip during shaving was also calculated from equation (6) and compared with the measured value. In this experiment, a cantilever with a force constant of $40 \mathrm{Nm}^{-1}$ was used. A force of $2000 \mathrm{nN}$ was employed during shaving. Young's modulus of bulk titanium dioxide is $282.76 \mathrm{GPa}$, and Poisson's ratio is 0.28 . Young's modulus of silicon nitride is $260 \mathrm{GPa}$, and Poisson's ratio is 0.26 . Then, from the relation given above (equation (6)), an applied force of $2000 \mathrm{nN}$ can result in a depression of 


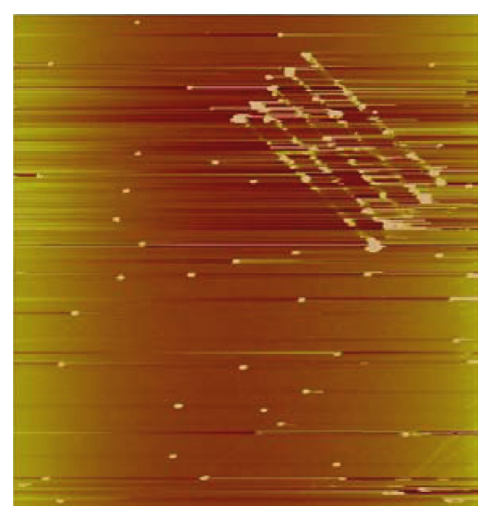

(a)

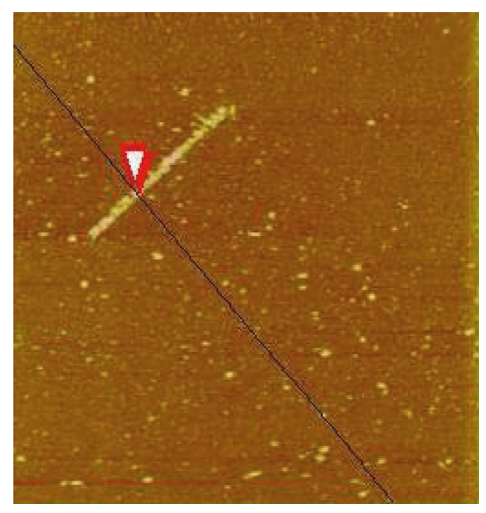

(b)

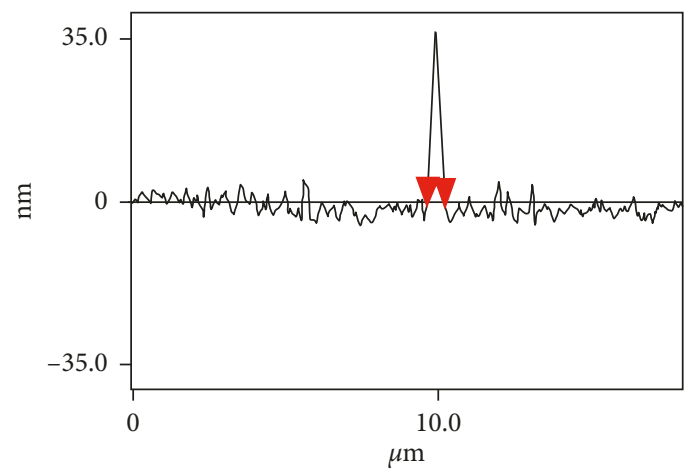

(c)

Figure 5: (a, b) $30 \times 30 \mu \mathrm{m}^{2}$ atomic force microscopy topographic images of NeutrAvidin-functionalized polystyrene nanoparticles attached to the shaved part of the monolayer. (c) Section analysis of (b).

$0.4 \mathrm{~nm}$ on the titanium oxide. Therefore, the calculation shows that there will be an indentation of $0.4 \mathrm{~nm}$ which is greater than the measured one, $0.2 \mathrm{~nm}$. The poly(ethylene glycol)-silane film was not considered during calculation. So, this may be the reason for the difference between the measured and the calculated value.

Site-specific attachment of nanoparticles was carried out by immersing the patterned samples into a solution that contains protein-coated nanoparticles. Figure 4(d) shows a topographic image of a NeutrAvidin-coated polystyrene nanoparticle attached to the shaved part of the poly(ethylene glycol)-silane monolayer formed on native oxide of titanium. As can be seen in Figures 5(a) and 5(b), the nanoparticles are attached exclusively onto the shaved part of the monolayer and the attachment to the unshaved part is minimum. The nonspecific attachment seen in Figure 5(a) is due to the low grafting density of the poly(ethylene glycol)silane monolayers on native oxide of titanium. This can be solved by increasing the immersion time, increasing the density of the hydroxyl groups on the surface by heating [19], and controlling the water content during monolayer formation. Enough water is required for all the silanes grafted on the substrate to hydrolyse and bond with each other. This increases the lateral network of the silanes which can prevent the penetration of the water molecules to the oxide surface [20]. The section analysis was done to study the size and aggregation of the nanoparticles attached to the shaved part. Figure 5(c) shows the section analysis of the topographic image of Figure 5(b). The section analysis shows that the height is ca $40 \mathrm{~nm}$. This demonstrates that the nanoparticles are only a monolayer thick.

\section{Conclusion}

Poly(ethylene glycol)-silane monolayers were deposited successfully on titanium oxide substrates. The atomic force microscopy and ellipsometry characterisation indicated that the monolayers are disordered and collapsed. After comparing the hydrated diameter with interchain distance, it was concluded that the monolayers fully covered the substrate. The poly(ethylene glycol)-silane monolayer has been patterned using atomic force microscopy and consequently used to pattern protein-coated nanoparticles. The section analysis showed that the height of the nanoparticle pattern is ca $40 \mathrm{~nm}$ which demonstrates that single particles are attached to the shaved part of the monolayer. This method can be used to pattern any type of protein-coated nanoparticles including noble metal nanoparticles.

\section{Data Availability}

The data used to support the findings of this study are available from the corresponding author upon request.

\section{Conflicts of Interest}

The author declares that there are no conflicts of interest regarding the publication of this paper.

\section{Acknowledgments}

The author acknowledges the Department of Chemistry, University of Sheffield, for financial support to carry out the experiments.

\section{References}

[1] J. C. Love, L. A. Estroff, J. K. Kriebel, R. G. Nuzzo, and G. M. Whitesides, "Self-assembled monolayers of thiolates on metals as a form of nanotechnology," Chemical Reviews, vol. 105, no. 4, pp. 1103-1170, 2005.

[2] A. Ulman, "Formation and structure of self-assembled monolayers," Chemical Reviews, vol. 96, no. 4, pp. 15331554, 1996.

[3] K. M. Mayer and J. H. Hafner, "Localized surface plasmon resonance sensors," Chemical Reviews, vol. 111, no. 6, pp. 3828-3857, 2011.

[4] F. Le, D. W. Brandl, Y. A. Urzhumov et al., "Metallic nanoparticle arrays: a common substrate for both surfaceenhanced Raman scattering and surface-enhanced infrared absorption," ACS Nano, vol. 2, no. 4, pp. 707-718, 2008.

[5] B. D. Gates, Q. Xu, J. C. Love, D. B. Wolfe, and G. M. Whitesides, "Unconventional nanofabrication," Annual Review of Materials Research, vol. 34, no. 1, pp. 339-372, 2004. 
[6] A. Kumar, H. A. Biebuyck, and G. M. Whitesides, "Patterning self-assembled monolayers: applications in materials science," Langmuir, vol. 10, no. 5, pp. 1498-1511, 1994.

[7] S. Sun and G. J. Leggett, "Micrometer and nanometer scale photopatterning of self-assembled monolayers of phosphonic acids on aluminum oxide," Nano Letters, vol. 7, no. 12, pp. 3753-3758, 2007.

[8] R. C. Dunn, "Near-field scanning optical microscopy," Chemical Reviews, vol. 99, no. 10, pp. 2891-2928, 1999.

[9] W. M. Wang, R. M. Stoltenberg, S. Liu, and Z. Bao, "Direct patterning of gold nanoparticles using dip-pen nanolithography," ACS Nano, vol. 2, no. 10, pp. 2135-2142, 2008.

[10] M. Schmudde, C. Grunewald, C. Goroncy et al., "Controlling the interaction and non-close-packed arrangement of nanoparticles on large areas," ACS Nano, vol. 10, no. 3, pp. 3525-3535, 2016.

[11] N. N. Khanh and K. B. Yoon, "Facile organization of colloidal particles into large, perfect one- and two-dimensional arrays by dry manual assembly on patterned substrates," Journal of the American Chemical Society, vol. 131, no. 40, pp. 1422814230, 2009.

[12] E. Mercado, S. Santiago, L. Baez et al., "One-dimensional silver nanostructures on single-wall carbon nanotubes," Nanoscale Research Letters, vol. 6, no. 1, p. 602, 2011.

[13] K. Wang, "The use of titanium for medical applications in the USA," Materials Science and Engineering: A, vol. 213, no. 1-2, pp. 134-137, 1996.

[14] A. Wörz, B. Berchtold, K. Moosmann, O. Prucker, and J. Rühe, "Protein-resistant polymer surfaces," Journal of Materials Chemistry, vol. 22, no. 37, article 19547, 2012.

[15] S. Chen, L. Li, C. Zhao, and J. Zheng, "Surface hydration: principles and applications toward low-fouling/nonfouling biomaterials," Polymer, vol. 51, no. 23, pp. 5283-5293, 2010.

[16] G. Tizazu, O. el Zubir, S. Patole et al., "Micrometer and nanometer scale photopatterning of proteins on glass surfaces by photo-degradation of films formed from oligo(ethylene glycol) terminated silanes," Biointerphases, vol. 7, no. 1, p. 54, 2012.

[17] J. N. Israelachvili and M. L. Gee, "Contact angles on chemically heterogeneous surfaces," Langmuir, vol. 5, no. 1, pp. 288-289, 1989.

[18] S. J. Sofia, V. Premnath, and E. W. Merrill, "Poly(ethylene oxide) grafted to silicon surfaces: grafting density and protein adsorption," Macromolecules, vol. 31, no. 15, pp. 5059-5070, 1998.

[19] R. W. Carpick and M. Salmeron, "Scratching the surface: fundamental investigations of tribology with atomic force microscopy," Chemical Reviews, vol. 97, no. 4, pp. 1163-1194, 1997.

[20] S. Belikov, S. Magonov, N. Erina et al., "Theoretical modelling and implementation of elastic modulus measurement at the nanoscale using atomic force microscope," Journal of Physics: Conference Series, vol. 61, pp. 1303-1307, 2007.

[21] J. A. Howarter and J. P. Youngblood, "Optimization of silica silanization by 3-aminopropyltriethoxysilane," Langmuir, vol. 22, no. 26, pp. 11142-11147, 2006.

[22] S. Sharma, R. W. Johnson, and T. A. Desai, "Evaluation of the stability of nonfouling ultrathin poly(ethylene glycol) films for silicon-based microdevices," Langmuir, vol. 20, no. 2, pp. 348-356, 2004.

[23] G. Tizazu, A. M. Adawi, G. J. Leggett, and D. G. Lidzey, "Photopatterning, etching, and derivatization of selfassembled monolayers of phosphonic acids on the native oxide of titanium," Langmuir, vol. 25, no. 18, pp. 1074610753, 2009.

[24] L. G. Rosa, J. Jiang, O. V. Lima et al., "Selective nanoshaving of self-assembled monolayers of 2-(4-pyridylethyl)triethoxysilane," Materials Letters, vol. 63, no. 12, pp. 961-964, 2009. 


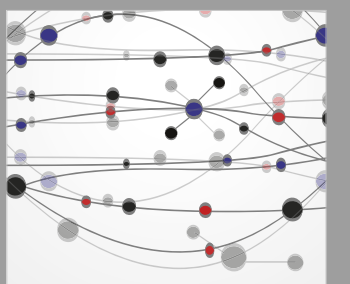

The Scientific World Journal
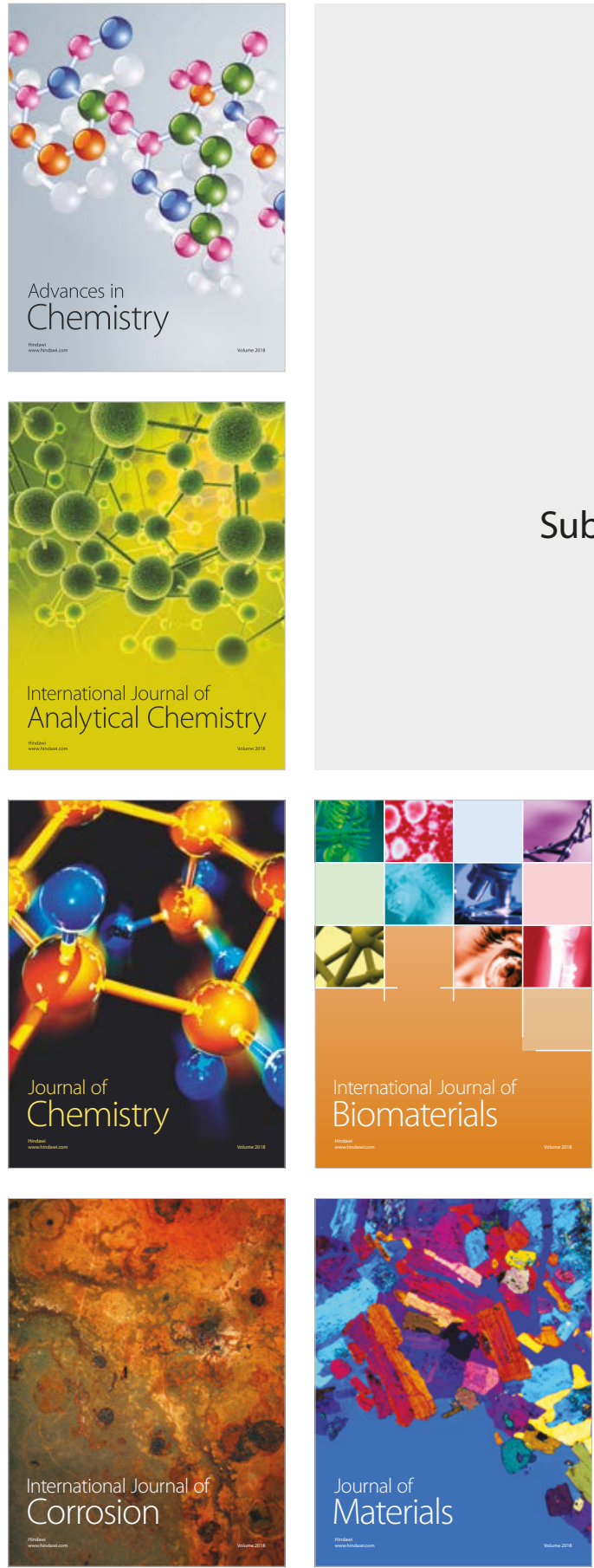

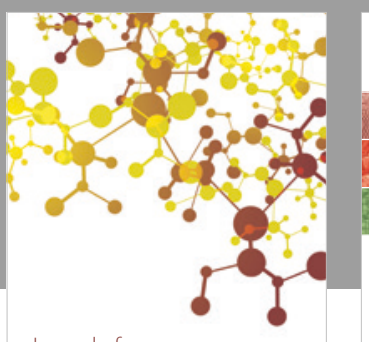

Journal of

Applied Chemistry
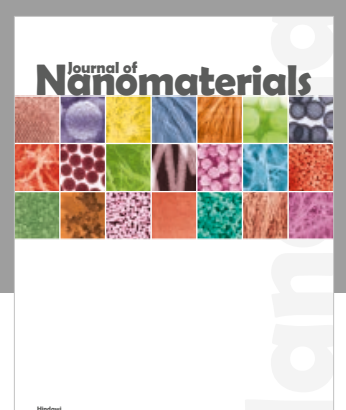

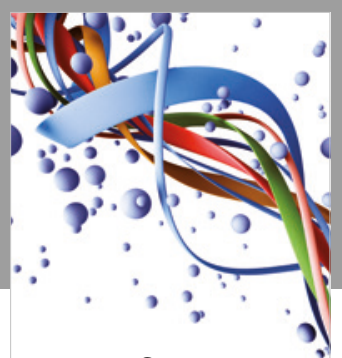

Scientifica

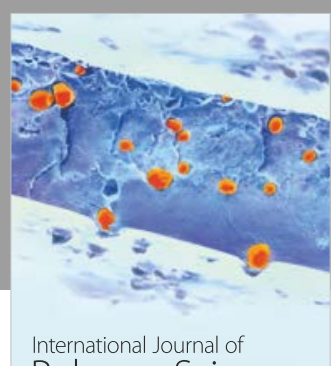

Polymer Science

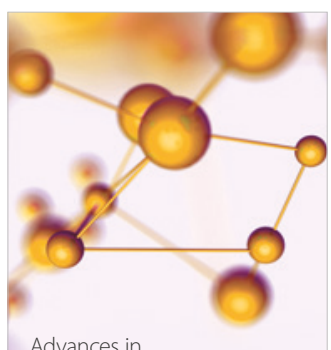

Physical Chemistry
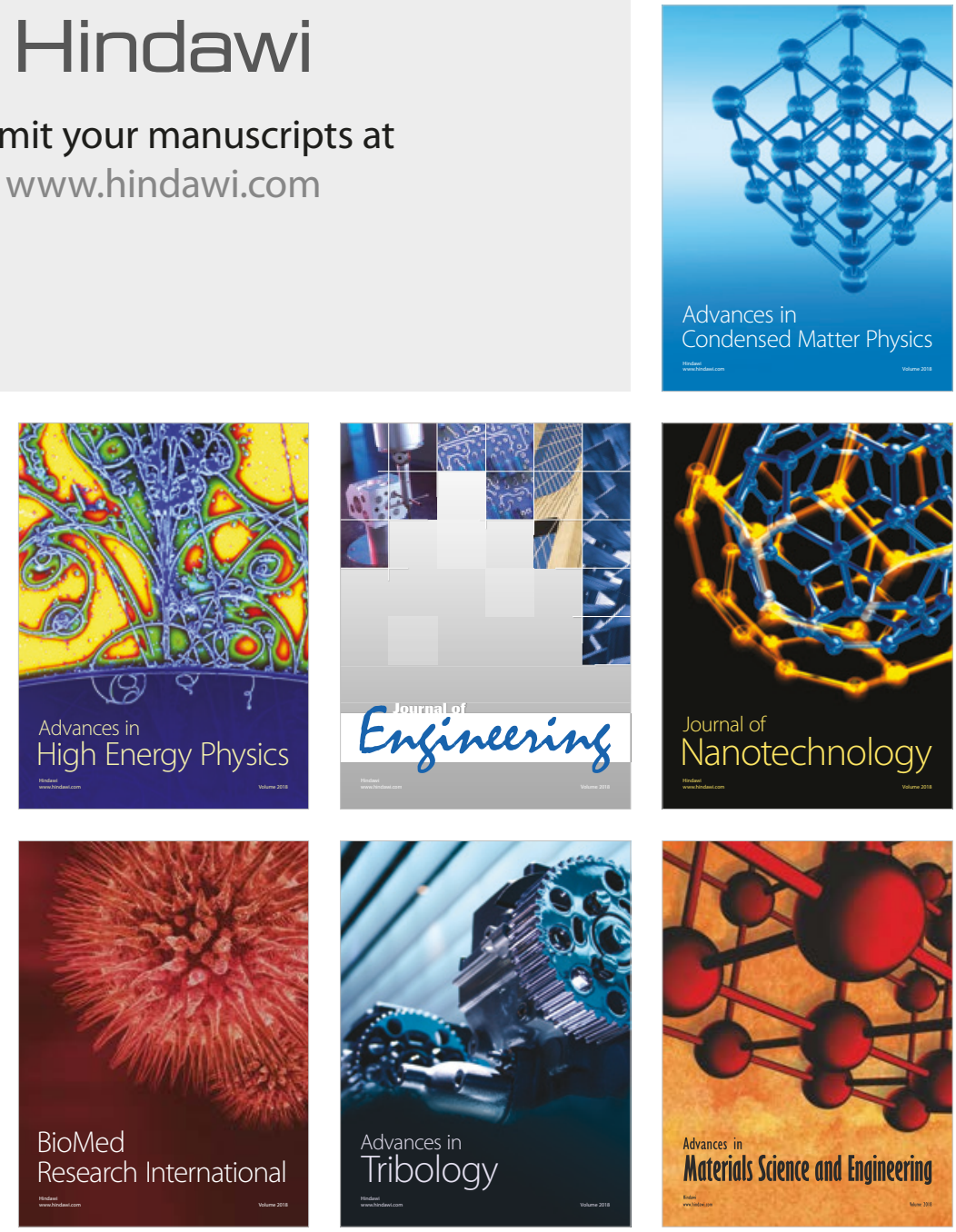